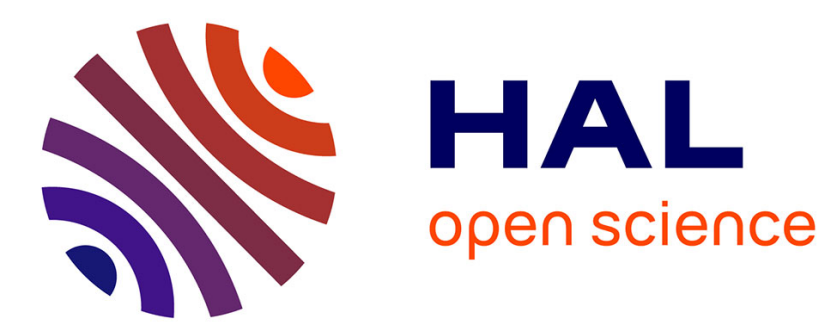

\title{
On stability radii in delay parameters
}

Wim Michiels, Emilia Fridman, Silviu-Iulian Niculescu

\section{To cite this version:}

Wim Michiels, Emilia Fridman, Silviu-Iulian Niculescu. On stability radii in delay parameters. 7th IFAC Workshop on Time Delay Systems TDS 2007, Sep 2007, Nantes, France. pp.322-329, 10.1016/S1474-6670(17)69308-X . hal-02293038

\section{HAL Id: hal-02293038 \\ https://hal.science/hal-02293038}

Submitted on 20 Sep 2019

HAL is a multi-disciplinary open access archive for the deposit and dissemination of scientific research documents, whether they are published or not. The documents may come from teaching and research institutions in France or abroad, or from public or private research centers.
L'archive ouverte pluridisciplinaire HAL, est destinée au dépôt et à la diffusion de documents scientifiques de niveau recherche, publiés ou non, émanant des établissements d'enseignement et de recherche français ou étrangers, des laboratoires publics ou privés. 


\title{
ON STABILITY RADII IN DELAY PARAMETERS
}

\author{
Wim Michiels * Emilia Fridman ** \\ Silviu-Iulian Niculescu ${ }^{* * *}$ \\ * Department of Computer Science, K.U.Leuven \\ Celestijnenlaan 200A, B-3001 Heverlee, Belgium \\ E-mail: Wim. Michiels@cs. kuleuven.be \\ and \\ Department of Mechanical Engineering, \\ Eindhoven University of Technology \\ PO Box 513, 5300 MB Eindhoven, the Netherlands \\ ** Department of Electrical Engineering, Tel-Aviv University, \\ Tel-Aviv, 69978, Israel \\ E-mail: emilia@eng.tau.ac.il \\ *** L2S (UMR CNRS 8506), CNRS-Supélec, \\ 3, rue Joliot Curie, 91192, Gif-sur-Yvette, France. \\ E-mail: Silviu.Niculescu@lss.supelec.fr
}

\begin{abstract}
This paper focuses on the robust stability analysis of a class of linear systems including multiple delays subjected to constant or time-varying perturbations. The approach considered makes use of appropriate stability radius concepts (dynamic, static) and relies on a feedback interconnection interpretation of the uncertain system. Various computable bounds on stability radii are obtained that exploit the structure of the problem. Systems including perturbations on both system matrices and delays are also dealt with.
\end{abstract}

Keywords: delays, uncertainty, stability radius, feedback interconnection.

\section{INTRODUCTION}

It is well-known that the characterization of stability regions in the parameter space for time-delay systems is a difficult problem (see, e.g. [Diekmann et al. (1995)], and the references therein). Furthermore, the characterization in the delay parameter space for linear systems with multiple delays is $\mathcal{N} \mathcal{P}$-hard as proven by [Toker and Özbay (1996)].

Stability radii are well known in the context of matrix distance problems, see Hinrichsen and Pritchard (2005) and the references therein. Recently, they have been used to assess or optimize robustness of stability of linear time-delay systems subjected to structured uncertainty on the corresponding system matrices in Michiels et al. (2006); Michiels and Roose (2003). In this context stability radii correspond to the size of the smallest perturbations that render the system unstable.

The aim of this paper is to adopt the concept of stability radii to linear systems including multiple delays subject to constant or time-varying perturbations on the delay parameters, and to derive computable expressions. The case of constant perturbations for a class of quasi-polynomials including two delays was addressed in a geometrical setting in [Gu et al. (2007)], where the authors introduced the notion of delay deviation. The idea can be resumed in computing the distance between the "nominal" point (in 
the delay parameter space) and the "closest" crossing curve for which there exists at least one characteristic root on the imaginary axis. Such a delay deviation characterization is nothing else than a characterization of a stability radius in the delay parameter space.

The approach considered in this paper is quite distinct to the one mentioned above. First, we will introduce two appropriate notions of stability radii: static and dynamic, in order to characterize constant and timevarying perturbations on the nominal system's parameters. These stability radii are scalar robustness measures based on an a priori chosen weighting of the perturbations of delays and -as we shall discuss at the end- system matrices. Secondly, we will employ a feedback interconnection interpretation of the uncertain system in order to derive estimates for the defined stability radii. Note that a similar point of view was taken in (Gu et al., 2003, Chapter 3); Kao and Lincoln (2004); Fridman and Gil' (2006); Fridman and Shaked (2006); Shustin and Fridman (2007) $\left(\mathcal{L}_{2}\right.$ gain analysis applied to systems with time-varying delay perturbations), Huang and Zhou (2000) ( $\mu$ analysis applied to systems with constant delay perturbations) and Michiels et al. (2006); Wagenknecht et al. (2007) (pseudospectra and stability radii for nonlinear eigenvalue problems), and some of the references therein. In the present paper the robust stability characterizations in these references are combined and extended in a unifying framework, and reformulated in terms of appropriately defined stability radii. Finally, in Kao and Rantzer (2007) and the references therein the IQC approach is applied to deal with time-varying delays, leading to easy-to-check stability conditions expressed as LMIs, under the assumption that the corresponding delay-free system is asymptotically stable. Such an assumption will not be made in this paper.

Although in the case of uncertainty on the delays the feedback interconnection point of view and related tools from robust control will typically lead to expressions for lower bounds on stability radii (corresponding to sufficient yet not necessary robust stability conditions), as we shall see, they offer several advantages. Explicit computable expressions for bounds are namely obtained that impose no limitations on the number of delays and the dimension of the problem. Also time-varying perturbations and combined perturbation on delays and system matrices (matrix valued perturbations) can be easily dealt with, as we shall demonstrate. Finally the interconnection framework is appropriate for solving associated synthesis problems. The latter issue will however not be further addressed in this paper.

The paper adopts a step-by-step approach, by imposing more conditions on the perturbations and exploiting this information accordingly. More precisely, first time-varying perturbations are considered in a $\mathcal{L}_{2}$ analysis framework. Next it is shown how the derived explicit bounds on the stability can be improved for the special case of constant perturbations, where besides the inherent increase of the stability radii (due to the restriction of the perturbations), the structure of the interconnection can be better exploited by using frequency domain techniques. Finally, implicit expressions are given which rely on exploiting all structure of the problem and leave conservatism only in the fact that phase information is not fully exploited in the feedback loop (inherent to the adopted approach). For reasons of simplicity and clarity of the presentation the cases of uncertainty on delays only and of uncertainty on both delays and system matrices are treated separately. The notations are standard.

\section{UNCERTAINTY ON THE DELAYS}

\subsection{Concept}

We address the uncertain system

$$
\begin{aligned}
& \dot{x}(t)=A_{0} x(t)+\sum_{i=1}^{m} A_{i} x\left(t-\tau_{i}-w_{i} \delta \tau_{i}(t)\right), \\
& x(\theta)=\phi(\theta),-\eta \leq \theta \leq 0, \phi \in \mathcal{C}\left([-\eta, 0], \mathbb{R}^{n}\right),
\end{aligned}
$$

where $x(t) \in \mathbb{R}^{n}, \eta>0, A_{i} \in \mathbb{R}^{n \times n}$ and $\tau_{i} \geq 0$. The uncertainty on the delays is modeled by uniformly bounded scalar functions $t \in[0, \infty] \rightarrow \delta \tau_{i}(t)$ and scalar weights $w_{i}>0$, which are such that

$$
w_{i} \delta \tau_{i}(t) \geq-\tau_{i}, \forall t \geq 0, \quad i=1, \ldots, m .
$$

We assume that the zero solution of the corresponding unperturbed system

$$
\begin{aligned}
& \dot{x}(t)=A_{0} x(t)+\sum_{i=1}^{m} A_{i} x\left(t-\tau_{i}\right), \\
& x(\theta)=\phi(\theta), \quad-\eta \leq \theta \leq 0,
\end{aligned}
$$

is asymptotically stable.

The dynamic stability radius $r_{\boldsymbol{\tau}}^{d}$ of the system (2) w.r.t. the delays is defined as:

$$
\begin{aligned}
& r_{\boldsymbol{\tau}}^{d}:=\sup \{\gamma \geq 0 \text { : the zero solution of }(1) \text { is } \\
& \text { asymptotically stable for all functions } \delta \boldsymbol{\tau}(t) \\
& \text { satisfying } \left.\sup _{t \geq 0}\left|\delta \tau_{i}(t)\right| \leq \gamma, i=1, \ldots, m\right\} .
\end{aligned}
$$

Note that, although $r_{\tau}^{d}$ explicitly depends on the weights $w_{i}$, this dependence is suppressed in the notation, for reasons of simplicity.

Similarly, if the uncertainty on the delay is assumed time-invariant then the static stability radius w.r.t. the delays is defined as:

$$
\begin{gathered}
r_{\boldsymbol{\tau}}^{s}:=\sup \{\gamma \geq 0: \text { the zero solution of }(1) \text { is } \\
\text { asymptotically stable for all constant } \\
\left.\delta \boldsymbol{\tau} \text { with }\left|\delta \tau_{i}\right| \leq \gamma, i=1, \ldots, m\right\} .
\end{gathered}
$$

In the next paragraph several lower bounds on the above stability radii are derived. Such lower bounds correspond to robust stability conditions. 


\subsection{Feedback interconnection point of view}

We factorize

$A_{i}=B_{i} C_{i}, B_{i} \in \mathbb{R}^{n \times n_{i}}, C_{i} \in \mathbb{R}^{n_{i} \times n}, i=1, \ldots, m$,

where all $B_{i}$ have full column rank, all $C_{i}$ have full row rank, and we let $\hat{n}=\sum_{i=1}^{m} n_{i}$.

For $u \in \mathcal{L}_{2}\left([0, \infty], \mathbb{R}^{\hat{n}}\right)$, let $y=\mathcal{G} u$ be defined by

$$
\left\{\begin{aligned}
\dot{x}(t) & =A_{0} x(t)+\sum_{i=1}^{m} A_{i} x\left(t-\tau_{i}\right) \\
& +\sum_{i=1}^{m}\left[B_{1} \cdots B_{m}\right] u(t), \quad x(\theta)=0, \theta \leq 0, \\
y(t) & =\left[w_{1} C_{1}^{T} \cdots w_{m} C_{m}^{T}\right]^{T} \dot{x}(t) .
\end{aligned}\right.
$$

Clearly $y \in \mathcal{L}_{2}\left([0, \infty], \mathbb{R}^{\hat{n}}\right)$. By the asymptotic stability of the unperturbed system and Parseval's theorem the $\mathcal{L}_{2}$-induced norm of $\mathcal{G}$ satisfies

where

$$
\|\mathcal{G}\|_{\mathcal{L}_{2}}=\|G(j \omega)\|_{\mathcal{H}_{\infty}}
$$

$$
\begin{gathered}
G(j \omega)=j \omega\left[\begin{array}{c}
w_{1} C_{1} \\
\vdots \\
w_{m} C_{m}
\end{array}\right] . \\
\left(j \omega I-A_{0}-\sum_{i=1}^{m} A_{i} e^{-j \omega \tau_{i}}\right)^{-1}\left[B_{1} \ldots B_{m}\right] .
\end{gathered}
$$

Next, we let

$$
\begin{aligned}
& \mathcal{S}_{i}^{\nu}: \mathcal{L}_{2}\left([0, \infty), \mathbb{R}^{\nu}\right) \mapsto \mathcal{L}_{2}\left([0, \infty), \mathbb{R}^{\nu}\right), \\
& \left(\mathcal{S}_{i}^{\nu} \xi\right)(t)=\frac{1}{w_{i}} \int_{t-\tau_{i}-w_{i} \delta \tau_{i}(t)}^{t-\tau_{i}} \tilde{\xi}(s) d s,
\end{aligned}
$$

where $\nu \in \mathbb{N}, i \in\{1, \ldots, m\}$, and $\tilde{\xi} \in \mathcal{L}_{2}\left(\mathbb{R}, \mathbb{R}^{\nu}\right)$ satisfies

$$
\tilde{\xi}(t)= \begin{cases}\xi(t), & t \geq 0 \\ 0, & t<0\end{cases}
$$

By defining

$$
\begin{aligned}
\mathcal{D} & : \mathcal{L}_{2}\left([0, \infty), \mathbb{R}^{\hat{n}}\right) \mapsto \mathcal{L}_{2}\left([0, \infty), \mathbb{R}^{\hat{n}}\right), \\
& (\mathcal{D} \xi)(t)=\operatorname{diag}\left(\left(\mathcal{S}_{1}^{n_{1}} \xi_{1}\right)(t), \ldots,\left(S_{m}^{n_{m}} \xi_{m}\right)(t)\right),
\end{aligned}
$$

where $\xi(t)=\left[\xi_{1}^{T}(t) \cdots \xi_{m}^{T}(t)\right]^{T}$, with $\xi_{i}(t) \in$ $\mathbb{R}^{n_{i}}, i=1, \ldots, m$, we can interpret the system (1) as a feedback interconnection of $\mathcal{G}$ and $\mathcal{D}$.

Remark 1. If some of the matrices $A_{i}, i=1, \ldots, m$, have low rank, then $\mathcal{G}$ and $\mathcal{D}$ have $\hat{n}<n m$ inputs and outputs, which is due to the factorization (5)

\subsection{Time-varying perturbations}

As a first step we characterize the induced $\mathcal{L}_{2}$ gain of $\mathcal{D}$. We need the following result from Shustin and Fridman $(2007,2006)$ :

Lemma 1. Assume that $\left|\delta \tau_{i}(t)\right| \leq \mu_{i}$ for all $t \geq$ 0 . Then the induced $\mathcal{L}_{2}$ norm of $\mathcal{S}_{i}^{\nu}$ is bounded by $\sqrt{7 / 4} \mu_{i}$.
Lemma 2. Assume that $\left|\delta \tau_{i}(t)\right| \leq \mu_{i}$ for all $t \geq 0$ and $1 \leq i \leq m$. Then

$$
\|\mathcal{D}\|_{\mathcal{L}_{2}} \leq \sqrt{7 / 4}\|\boldsymbol{\mu}\|_{\infty}
$$

Proof. Expression (9) follows from

$$
\|\mathcal{D}\|_{\mathcal{L}_{2}}=\max _{1 \leq i \leq m}\left\|\mathcal{S}_{i}^{n_{i}}\right\|_{\mathcal{L}_{2}}
$$

and Lemma 1.

By combining the above lemmas we arrive at the main result of this section:

Proposition 1. We have the following estimate:

$$
r_{\tau}^{d} \geq \frac{1}{\sqrt{7 / 4}}\left(\|G(j \omega)\|_{\mathcal{H}_{\infty}}\right)^{-1} .
$$

Proof. From the small gain theorem we have that if

$$
\|\mathcal{G}\|_{\mathcal{L}_{2}}\|\mathcal{D}\|_{\mathcal{L}_{2}}=\|G(j \omega)\|_{\mathcal{H}_{\infty}}\|\mathcal{D}\|_{\mathcal{L}_{2}}<1
$$

then the feedback interconnection of $\mathcal{G}$ and $\mathcal{D}$ is $\mathcal{L}_{2^{-}}$ stable, which induces the asymptotic stability of the zero solution of (1). Under the assumptions of Lemma 2 the condition (11) is fulfilled if

$$
\sqrt{7 / 4}\|G(j \omega)\|_{\mathcal{H}_{\infty}}\|\boldsymbol{\mu}\|_{\infty}<1 .
$$

The assertion of the proposition follows.

Proposition 1 can be strengthened by an appropriate scaling in the feedback loop. More precisely, with the set $\mathcal{T}$ defined as

$$
\begin{aligned}
\mathcal{T}= & \left\{\operatorname{diag}\left(T_{1}, \ldots, T_{m}\right): T_{i} \in \mathbb{C}^{n_{i} \times n_{i}},\right. \\
& \left.\operatorname{det} T_{i} \neq 0, i=1, \ldots, m\right\},
\end{aligned}
$$

we get:

Proposition 2. We have the following estimate:

$$
r_{\tau}^{d} \geq \frac{1}{\sqrt{7 / 4}}\left(\min _{T \in \mathcal{T}}\left\|T G(j \omega) T^{-1}\right\|_{\mathcal{H}_{\infty}}\right)^{-1} .
$$

Remark 2. The optimization problem

$$
\min _{T \in \mathcal{T}}\left\|T G(j \omega) T^{-1}\right\|_{\mathcal{H}_{\infty}}
$$

can be reformulated as

$$
\begin{gathered}
\min _{U, \gamma} \gamma, \\
\text { such that }
\end{gathered}
$$$$
\gamma>0, U \in \mathcal{T}, U=U^{*}>0,
$$$$
G(j \omega)^{*} U G(j \omega)-\gamma^{2} U<0, \forall \omega \geq 0,
$$

where $T$ can be computed from $U=T^{*} T$. Hence, a good scaling matrix $T$ in (14) can for instance be obtained by relaxing (15) to values of $\omega$ on a frequency grid and solving the resulting convex optimization problem. 
Remark 3. If the delay perturbations are such that the functions

$$
t \mapsto t-\tau_{i}-w_{i} \delta \tau_{i}(t), \quad i=1, \ldots, m,
$$

are non-decreasing, then the factor $\sqrt{7 / 4}$ in (10) and (14) can be replaced with 1 . This follows from the fact that in such case $\left\|S_{i}^{\nu}\right\|_{\mathcal{L}_{2}} \leq \mu_{i}$ if $\left|\delta \tau_{i}(t)\right| \leq \mu_{i}$ for all $t \geq 0$, see Fridman and Shaked (2006).

For improvements of Lemma 1 for the case where the delays are differential functions with a given upper bound on their derivatives, we refer to Shustin and Fridman (2006).

\subsection{Time-invariant perturbations}

We reconsider the estimates for the stability radii under the additional assumption of constant delay perturbations. Then improvements can be made by decoupling the signals in the frequency domain, and by further exploiting the structure in the problem under consideration.

Let the entire functions $s_{i}$ be defined as

$$
\begin{aligned}
s_{i}(\lambda) & =\left\{\begin{array}{ll}
e^{-\lambda \tau_{i}} \frac{1-e^{-\lambda\left(w_{i} \delta \tau_{i}\right)}}{w_{i} \lambda}, & \lambda \neq 0 \\
1, & \lambda=0
\end{array},\right. \\
i & =1, \ldots, m .
\end{aligned}
$$

As they satisfy

$$
\begin{gathered}
\left|s_{i}(j \omega)\right| \leq\left|\frac{1-e^{-j \omega\left(w_{i} \delta \tau_{i}\right)}}{w_{i} \omega}\right| \leq\left|\frac{\sin \frac{w_{i} \delta \tau_{i}}{2} \omega}{\frac{w_{i}}{2} \omega}\right| \leq \delta \tau_{i}, \\
\forall \omega \geq 0
\end{gathered}
$$

we obtain

$$
\left\|S_{i}^{\nu}\right\|_{\mathcal{L}_{2}}=\left\|s_{i}(j \omega) I_{\nu}\right\|_{\mathcal{H}_{\infty}} \leq \delta \tau_{i}
$$

This result can also be derived in the time-domain, see [Fridman and Shaked (2006)]. Denote with

$$
D(\lambda):=\operatorname{diag}\left(s_{1}(\lambda) I_{n_{1}}, \ldots, s_{m}(\lambda) I_{n_{m}}\right)
$$

transfer function associated with the operator $\mathcal{D}$, defined in (8). From (16) it follows that

$$
\|D(j \omega)\|_{\mathcal{H}_{\infty}} \leq \delta \tau .
$$

The characteristic equation of (1) can be written on the imaginary axis as

$$
\begin{array}{r}
\operatorname{det}\left(j \omega I-A_{0}-\sum_{i=1}^{m} A_{i} e^{-j \omega \tau_{i}}\right) . \\
\cdot \operatorname{det}(I-G(j \omega) D(j \omega))=0,
\end{array}
$$

where the first factor is nonzero for all $\omega \geq 0$ because the unperturbed system is assumed to be asymptotically stable. The perturbed system is asymptotically stable if the perturbations cannot shift characteristic roots to the imaginary axis, that is, if (18) has no solutions. Based on this observation we have the following result, which makes use of structured singular values (see the appendix for a short introduction):

Proposition 3. Define the uncertainty set

$\boldsymbol{\Delta}:=\left\{\operatorname{diag}\left(d_{1} I_{n_{1}}, \ldots, d_{m} I_{n_{m}}\right): d_{i} \in \mathbb{C}, 1 \leq i \leq m\right\}$.

Then

$$
r_{\tau}^{s} \geq\left(\sup _{\omega \geq 0} \mu_{\Delta} G(j \omega)\right)^{-1}
$$

where $\mu_{\Delta}(\cdot)$ is the structured singular value w.r.t. (19).

Proof. From (18) and the fact that $D(j \omega) \in \boldsymbol{\Delta}$ for all $\omega \geq 0$, a sufficient stability condition is given by

$$
\|D(j \omega)\|_{2}<\frac{1}{\mu_{\Delta}(G(j \omega))}, \forall \omega \geq 0 .
$$

This condition is satisfied if

$$
\|\boldsymbol{\delta} \tau\|_{\infty}<\left(\mu_{\boldsymbol{\Delta}}(G(j \omega))\right)^{-1}, \forall \omega \geq 0
$$

which leads to the statement of the proposition.

Because the exact computation of the structured singular of a complex $\hat{n} \times \hat{n}$ matrix $M$ w.r.t. the uncertainty structure (19) is a hard problem if $m$ is large [Toker and Özbay (1995)], the available numerical algorithms typically compute lower and upper bounds, see the appendix. We have for instance

$$
\mu_{\Delta}(M) \leq \min _{T \in \mathcal{T}} \sigma_{1}\left(T M T^{-1}\right),
$$

where $\mathcal{T}$ is given by (13) and $\sigma_{1}(\cdot)=\|\cdot\|_{2}$. The computation of the upper bound in (21) can be formulated as a convex optimization problem, using the arguments spelled out in Remark 2.

From Proposition 3 and the estimate (21) we obtain:

$$
r_{\tau}^{s} \geq\left(\sup _{\omega \geq 0} \min _{T \in \mathcal{T}}\left\|T^{-1} G(j \omega) T\right\|_{2}\right)^{-1} .
$$

It is instructive to compare expressions (22) and (14), the latter corresponding to:

$$
r_{\tau}^{d} \geq\left(\sqrt{7 / 4} \min _{T \in \mathcal{T}} \sup _{\omega \geq 0}\left\|T^{-1} G(j \omega) T\right\|_{2}\right)^{-1} .
$$

Besides the factor $\sqrt{7 / 4}$ (due to the better estimate of $\left\|S_{i}^{\nu}\right\|_{\mathcal{L}_{2}}$ in the time-invariant case), the outer and inner optimization have been inter-changed, that is, the scaling has become frequency dependent in (22).

Further improvements of the estimate (20) can be made by using the smallest possible upper bound on $\left|s_{i}(j \omega)\right|$ instead of (16). The price to be paid is that the expression for the stability radius is no longer explicit. The following result slightly generalizes Theorem 3 of Huang and Zhou (2000): 
Proposition 4. Let $s: \mathbb{R}_{+} \rightarrow \mathbb{R}_{+}$,

$$
\omega \mapsto s(\omega):= \begin{cases}\sin (\omega), & \omega \leq \frac{\pi}{2} \\ 1, & \omega \geq \frac{\pi}{2} .\end{cases}
$$

Define $F: \mathbb{R}_{+} \backslash\{0\} \rightarrow \mathbb{R}_{+}$,

$$
\begin{aligned}
\alpha \rightarrow F(\alpha):= & \sup _{\omega \geq 0} \mu_{\Delta}\left(\left[\begin{array}{c}
2 s\left(\frac{w_{1} \alpha \omega}{2}\right) C_{1} \\
\vdots \\
2 s\left(\frac{w_{m} \alpha \omega}{2}\right) C_{m}
\end{array}\right]^{-1} .\right. \\
& \cdot\left(j \omega I-A_{0}-\sum_{i=1}^{m} A_{i} e^{-j \omega \tau_{i}}\right)^{-1} \\
& \left.\cdot\left[B_{1} \ldots B_{m}\right]\right),
\end{aligned}
$$

where $\mu_{\Delta}(\cdot)$ is the structured singular value w.r.t. the uncertainty set (19).

If $F(\alpha)<1$, then $r_{\tau}^{s} \geq \alpha$. Consequently,

$$
r_{\boldsymbol{\tau}}^{s} \geq \sup \{\alpha>0: F(\alpha)<1\} .
$$

Proof. The proof is based on an additional scaling within the feedback loop. Equation (18) is equivalent with

$$
\operatorname{det}\left(I-\Lambda^{-1}(\omega ; \alpha) G(j \omega) D(j \omega) \Lambda(\omega ; \alpha)\right)=0,
$$

where

$$
\Lambda(\omega ; \alpha)=\operatorname{diag}\left(\frac{j \omega w_{1}}{2 s\left(\frac{w_{1} \alpha \omega}{2}\right)} I_{n_{1}}, \ldots, \frac{j \omega w_{m}}{2 s\left(\frac{w_{1} \alpha \omega}{2}\right)} I_{n_{m}}\right) .
$$

By construction, we have

$$
F(\alpha)=\sup _{\omega \geq 0} \mu_{\boldsymbol{\Delta}}\left(\Lambda^{-1}(\omega ; \alpha) G(j \omega)\right) .
$$

Furthermore, the structure of $D(j \omega)$ is not affected by the post-multiplication with $\Lambda(\omega ; \alpha)$. Hence, under the assumption $F(\alpha)<1$, the system is stable if

$$
\begin{aligned}
& \|\Lambda(\omega ; \alpha) D(j \omega)\|_{2}<1, \forall \omega \geq 0 \\
& \Leftrightarrow\left|\frac{1-e^{-j \omega w_{i} \delta \tau_{i}}}{2 s\left(\frac{w_{i} \alpha \omega}{2}\right)}\right|<1, \forall \omega \geq 0, i=1, \ldots, m \\
& \Leftrightarrow\left|\frac{\sin \left(\frac{w_{i} \delta \tau_{i} \omega}{2}\right)}{s\left(\frac{w_{i} \alpha \omega}{2}\right)}\right|<1, \forall \omega \geq 0, i=1, \ldots, m \\
& \Leftrightarrow\left|\delta \tau_{i}\right|<\alpha, i=1, \ldots, m .
\end{aligned}
$$

The assertion of the proposition follows.

Remark 4. Since for all $\omega \geq 1$ and $i=1, \ldots, m$, we have

$$
\sup _{\left|\delta \tau_{i}\right|<\alpha}\left|\frac{\sin \left(\frac{w_{i} \delta \tau_{i} \omega}{2}\right)}{s\left(\frac{w_{i} \alpha \omega}{2}\right)}\right|=1, \quad \forall \omega \geq 0,
$$

a further improvement of the estimate (25) can only be achieved by exploiting phase information in the feedback loop, which is not possible with the adopted $\mu$ approach.

\section{UNCERTAINTY IN COEFFICIENT MATRICES AND DELAYS}

We consider the uncertain system

$$
\begin{aligned}
& \dot{x}(t)=\left(A_{0}+D_{0} \delta A_{0}(t) E_{0}\right) x(t) \\
& +\sum_{i=1}^{m}\left(A_{i}+D_{i} \delta A_{i}(t) E_{i}\right) x\left(t-\tau_{i}-w_{i} \delta \tau_{i}(t)\right),
\end{aligned}
$$

under appropriate initial conditions. The uncertainty is expressed by the piece-wise continuous functions

$$
\begin{aligned}
& \delta A_{i} \in \mathcal{L}_{\infty}\left([0, \infty), \mathbb{R}^{n_{i} \times n_{i}}\right), \quad i=0, \ldots, m, \\
& \delta \tau_{i} \in \mathcal{L}_{\infty}\left([0, \infty),\left[-\tau_{i}, \infty\right)\right), i=1, \ldots, m,
\end{aligned}
$$

while $D_{i} \in \mathbb{R}^{n \times n_{i}}$ and $E_{i} \in \mathbb{R}^{n_{i} \times n}$ are weight matrices, and $w_{i}>0$ are scalar weights.

The dynamic stability radius of the unperturbed system (2) w.r.t. the combined uncertainty in (26) is defined as

$r_{c}^{d}=: \sup \{\gamma \geq 0:$ the zero solution of $(26)$ is asymptotically stable for all functions $\delta A_{i}(t)$ and $\delta \boldsymbol{\tau}(t)$ with ess sup $\left\|\delta A_{i}(t)\right\|_{2} \leq \gamma, i=0, \ldots, m$ and $\left.\operatorname{ess}_{\sup _{t \geq 0}}\left|\delta \tau_{i}(t)\right| \leq \gamma, i=1, \ldots, m\right\}$.

The corresponding static stability radius $r_{c}^{s}$ is defined in a similar way, by assuming time-invariant perturbations.

From an analysis point of view the main difference w.r.t. case discussed in the previous section is the nonlinear dependence of the righthand side of (26) on the uncertainty, in particular, on the products of $\delta A_{i}$ and $x\left(t-\tau_{i}-w_{i} \delta \tau_{i}\right)$. This problem can be overcome by introducing additional input and outputs [Fridman and Shaked (2006)]. First, let $B_{i}, C_{i}, \tilde{A}_{i}, \tilde{D}_{i}, \tilde{E}_{i}$ be such that

$$
\begin{gathered}
A_{i}+D_{i} \delta A_{i}(t) E_{i}=B_{i}\left(\tilde{A}_{i}+\tilde{D}_{i} \delta A_{i}(t) \tilde{E}_{i}\right) C_{i} \\
i=1, \ldots, m
\end{gathered}
$$

where each $C_{i} \in \mathbb{R}^{\tilde{n}_{i} \times n}$ has full row rank. A trivial choice is given by

$$
\begin{aligned}
& B_{i}=C_{i}=I, \quad \tilde{A}_{i}=A_{i}, \quad \tilde{D}_{i}=D_{i}, \\
& \tilde{E}_{i}=E_{i}, \quad i=1, \ldots, m,
\end{aligned}
$$

yet it is beneficial if a decomposition can be chosen where $\operatorname{rank}\left(C_{i}\right)=\tilde{n}_{i}<n$ (as this leads to smaller block sizes in the uncertainty structure). Next, we write (26) as the feedback interconnection of the system 


$$
\left\{\begin{aligned}
\dot{x}(t) & =A_{0} x(t)+\sum_{i=1}^{m} A_{i} x\left(t-\tau_{i}\right) \\
& +D_{0} \tilde{u}_{0}(t)+\sum_{i=1}^{m} B_{i} \tilde{D}_{i} \tilde{u}_{i}(t)+\sum_{i=1}^{m} B_{i} \tilde{A}_{i} u_{i}(t), \\
\tilde{y}_{0}(t) & =E_{0} x(t), \\
\tilde{y}_{i}(t) & =\tilde{E}_{i} C_{i} x\left(t-\tau_{i}\right)+\tilde{E}_{i} u_{i}, i=1, \ldots, m \\
y_{i}(t) & =-\zeta w_{i} C_{i} \dot{x}(t), \quad i=1, \ldots, m
\end{aligned}\right.
$$

where $\zeta>0$ and the control loop is closed with

$$
\begin{cases}\tilde{u}_{i}(t)=\delta A_{i}(t) \tilde{y}_{i}(t), & i=0, \ldots, m, \\ u_{i}(t)=\frac{1}{\zeta}\left(\mathcal{S}_{I}^{\tilde{n}_{i}} y_{i}\right)(t), & i=1, \ldots, m .\end{cases}
$$

Using this feedback interconnection point of view, lower bounds on the stability radii can be derived analogously as in the case where only the delays are uncertain, which we have discussed in the previous section. In the sequel, we therefore restrict ourselves to formulating the main results.

Remark 5. The nonlinear dependence of the right hand side of (26) on the uncertainty can alternatively be removed by a transformation to a descriptor system. Such an approach is proposed in (Wagenknecht et al., 2007, Section 2.1)

\subsection{Time-varying perturbations}

Let $G$ be the transfer function of (29), that is,

$$
\begin{aligned}
& G(\lambda ; \zeta)=\left[\begin{array}{c}
E_{0} \\
e^{-\lambda \tau_{1}} \tilde{E}_{1} C_{1} \\
\vdots \\
e^{-\lambda \tau_{m}} \tilde{E}_{m} C_{m} \\
-\zeta w_{1} \lambda C_{1} \\
\vdots \\
-\zeta w_{m} \lambda C_{m}
\end{array}\right] . \\
& {\left[\lambda I-A_{0}-\sum_{i=1}^{m} A_{i} e^{-\lambda \tau_{i}}\right]^{-1} .} \\
& {\left[D_{0} B_{1} \tilde{D}_{1} \cdots B_{m} \tilde{D}_{m} B_{1} \tilde{A}_{1} \cdots B_{m} \tilde{A}_{m}\right]+} \\
& {\left[\begin{array}{cccccc}
0 & \cdots & 0 & & & 0 \\
& & 0 & \tilde{E}_{1} & & \\
& & & & \ddots & \\
& & & & & \tilde{E}_{m} \\
0 & & & & & 0 \\
\vdots & & & & & \vdots \\
0 & & & & \cdots & 0
\end{array}\right] .}
\end{aligned}
$$

Proposition 5. We have the following estimate:

$$
r_{c}^{d} \geq\left(\|G(j \omega ; \sqrt{7 / 4})\|_{\mathcal{H}_{\infty}}\right)^{-1} .
$$

This proposition can again be strengthened by an appropriate scaling in the feedback loop. With the set $\mathcal{T}$ defined as

$$
\begin{array}{r}
\mathcal{T}=\left\{\operatorname{diag}\left(t_{0} I_{n_{0}}, \ldots, t_{m} I_{n_{m}}, T_{1}, \ldots, T_{m}\right):\right. \\
t_{i}>0, i=0, \ldots, m, T_{i} \in \mathbb{C}^{\tilde{n}_{i} \times \tilde{n}_{i}}, \\
\left.\operatorname{det} T_{i} \neq 0, i=1, \ldots, m\right\},
\end{array}
$$

we obtain:

$$
r_{c}^{d} \geq\left(\min _{T \in \mathcal{T}}\left\|T^{-1} G(j \omega ; \sqrt{7 / 4}) T\right\|_{\mathcal{H}_{\infty}}\right)^{-1} .
$$

\subsection{Time-invariant perturbations}

Taking into account the structure of the feedback path (30) and the estimate (17), we arrive at:

Proposition 6. Define the uncertainty set

$$
\begin{array}{r}
\Delta:=\left\{\operatorname{diag}\left(\Delta_{0}, \ldots, \Delta_{m}, d_{1} I_{\tilde{n}_{1}}, \ldots, d_{m} I_{\tilde{n}_{m}}\right):\right. \\
\Delta_{i} \in \mathbb{C}^{n_{i} \times n_{i}}, d_{j} \in \mathbb{C}, \\
i=0, \ldots, m, 1 \leq j \leq m\} .
\end{array}
$$

Then

$$
r_{c}^{s} \geq\left(\sup _{\omega \geq 0} \mu_{\Delta}(G(j \omega ; 1))\right)^{-1} .
$$

Using the scaling based upper bound on the structured singular value, described in the appendix, we arrive at:

$$
r_{c}^{s} \geq\left(\sup _{\omega \geq 0} \min _{T \in \mathcal{T}}\left\|T^{-1} G(j \omega ; 1) T\right\|_{2}\right)^{-1},
$$

where $\mathcal{T}$ is given by (32).

An improvement of estimate (17) finally leads to:

Proposition 7. Let the function $s: \mathbb{R}_{+} \rightarrow \mathbb{R}_{+}$be given by (23). Define $F: \mathbb{R}_{+} \backslash\{0\} \rightarrow \mathbb{R}_{+}$,

$$
\alpha \mapsto F(\alpha):=\sup _{\omega \geq 0} \mu_{\boldsymbol{\Delta}}\left(G_{2}(j \omega ; \alpha)\right),
$$

where

$$
\begin{aligned}
& G_{2}(\lambda ; \alpha)=\left[\begin{array}{c}
\alpha E_{0} \\
e^{-\lambda \tau_{1}} \alpha \tilde{E}_{1} C_{1} \\
\vdots \\
e^{-\lambda \tau_{m}} \alpha \tilde{E}_{m} C_{m} \\
-2 s\left(\frac{w_{1} \alpha \omega}{2}\right) C_{1} \\
\vdots \\
-2 s\left(\frac{w_{m} \alpha \omega}{2}\right) C_{m}
\end{array}\right] . \\
& {\left[\lambda I-A_{0}-\sum_{i=1}^{m} A_{i} e^{-\lambda \tau_{i}}\right]^{-1} \text {. }} \\
& {\left[\begin{array}{lll}
D_{0} & B_{1} \tilde{D}_{1} \cdots B_{m} \tilde{D}_{m} & B_{1} \tilde{A}_{1} \cdots B_{m} \tilde{A}_{m}
\end{array}\right]+} \\
& {\left[\begin{array}{ccccccc}
0 & \cdots & 0 & & & & 0 \\
& & 0 & \alpha \tilde{E}_{1} & & \\
& & & & \ddots & \\
& & & & & \alpha \tilde{E}_{m} \\
& & & & & 0 \\
0 & & & & & 0 \\
\vdots & & & & & \vdots \\
0 & & & & \cdots & 0
\end{array}\right] .}
\end{aligned}
$$

If $F(\alpha) \leq 1$, then $r_{c}^{s} \geq \alpha$. Consequently,

$$
r_{c}^{s} \geq \sup \{\alpha>0: F(\alpha)<1\} .
$$




\section{CONCLUDING REMARKS}

Stability radii of uncertain time-delay were defined and lower bounds were derived using a feedback interconnection point of view. Both constant and timevarying perturbations were considered on the delays, as well as delays and system matrices.

If information on the delays' variation and / or derivatives is available, then the derived estimates for the dynamic stability radii may be further improved, as we indicated in Remark 3.

Alternative estimates for the stability radii can be obtained by rewriting the uncertain system as a feedback interconnection, where the operator $\mathcal{D}_{2}$ or $\mathcal{D}_{3}$,

$$
\begin{aligned}
& \mathcal{D}_{2}(\eta)(t)=\left[\left(\mathcal{S}_{1}^{n_{1}} \eta\right)(t) \cdots\left(S_{m}^{n_{m}} \eta\right)(t)\right]^{T}, \\
& \mathcal{D}_{3}(\zeta)(t)=\sum_{k=1}^{m}\left(\mathcal{S}_{k}^{n_{k}} \zeta_{k}\right)(t),
\end{aligned}
$$

appears in the feedback loop instead of $\mathcal{D}$. For particular cases, e.g. scalar systems with multiple timevarying delays, this may lead to improved bounds. This is currently under investigation.

\section{ACKNOWLEDGEMENTS}

This paper present results of the Belgian Programme on Interuniversity Poles of Attraction, initiated by the Belgian State, Prime Ministers Office for Science, Technology and Culture, and of the Center of Excellence on Optimization in Engineering of the K.U.Leuven. Wim Michiels is a postdoctoral fellow of the Fund for Scientific Research -Flanders (Belgium).

\section{REFERENCES}

Diekmann, O., van Gils, S. A., Verduyn-Lunel, S. M. and Walther, H. -O. (1995). Delay equations, Functional-, Complex and Nonlinear Analysis (Appl. Math. Sciences Series, vol. 110, SpringerVerlag: New York).

Fridman, E. and Gil, M. (2007). Stability of linear systems with time-varying delays: A direct frequency domain approach, Journal of Computational and Applied Mathematics, vol. 200, pp. 61-66.

Fridman, E. and Shaked, U. (2006). Input-output approach to stability and $L_{2}$-gain analysis of systems with time-varying delays, Systems and Control Letters, vol. 55, pp. 1041-1063.

Gu, K., Kharitonov, V. L. and Chen, J. (2003). Stability of time-delay systems (Birkhäuser).

Gu, K., Niculescu, S.-I. and Chen, J. (2007). Computing maximum delay deviation allowed to retain stability in systems with two delays. in Applications of time-delay systems (J. Chiasson, J.-J. Loiseau, Eds.), Springer-Verlag: Heidelberg, LNCIS, vol. 352.
Hinrichsen, D. and Pritchard, A. J. (2005). Mathematical systems theory I. Modelling, state space analysis, stability and robustness (Springer-Verlag,Texts in Applied Mathematics, vol. 48).

Huang, Y.-P. and Zhou, K. (2000). Robust stability of uncertain time-delay systems. IEEE Transactions on Automatic Control, vol. 45, pp. 2169-2173.

Kao, C.-Y. and Lincoln, B. (2004). Simple stability criteria for systems with time-varying delays Automatica, vol. 40, pp. 1429-1434.

Kao, C.-Y. and Rantzer, A. (2007). Stability analysis of systems with uncertain time-varying delays $A u$ tomatica, vol. 43, pp. 959-970.

Michiels, W., Green, K., Wagenknecht, T. and Niculescu, S.-I. (2006). Pseudospectra and stability radii for analytic matrix functions with application to time-delay systems, Linear Algebra and its Applications, vol. 418, pp. 315-335.

Michiels, W., Plomteux, T. and Roose, D. (2005). Robust stabilization of linear time delay systems via the optimization of real stability radii, Proceedings of the 16th IFAC World Congress, Prague, the Czech Republic.

Michiels, W. and Roose, D. (2003). An eigenvalue based approach for the robust stabilization of linear time-delay systems, International Journal of Control, vol. 76, pp. 678-686.

Packard, A., Fan, M. and Doyle, J. C. (1988). A power method for the structured singular value, Proceedings of the 27th IEEE Conference on Control and Decision, Austin, USA, pp. 2132-2137.

Packard, A. and Doyle, J. C. (1993). The complex structured singular value, Automatica, vol. 29, pp. 71-109.

Shustin, E. and Fridman, E. (2007). On delay derivative-dependent control of systems with fast varying delays, Automatica, to appear.

Shustin, E. and Fridman, E. (2006). An improved stability analysis of linear systems with fast-varying delays, Proceedings of the 5th IFAC Symposium on Robust Control Design, Toulouse, France.

Toker, O. and Özbay, H. (1995). On the $\mathcal{N} \mathcal{P}$ hardness of the purely complex $\mu$ computation, analysis/synthesis, and some related problems in multidimensional systems, Proceedings of the 1995 American Control Conference, Seattle, USA, pp. 447-451.

Toker, O. and Özbay, H. (1996). Complexity issues in robust stability of linear delay-differential systems. Mathematics of Control, Signals, and Systems, vol. 9, pp. 386-400.

Wagenknecht, T., Michiels, W. and Green, K. (2007). Structured pseudospectra for nonlinear eigenvalue problems. Journal of Computational and Applied Mathematics. In press (available online).

Zhou, K., Doyle, J. C. and Glover, K. (1995). Robust and optimal control. (Prentice Hall). 


\section{Appendix A. THE STRUCTURED SINGULAR VALUE}

We introduce the concept of structured singular values of matrices and outline the main principles behind the standard computational schemes. A more elaborate introduction can be found in the review paper [Packard and Doyle (1993)], Chapter 11 of [Zhou et al. (1995)] and Chapter 4 of [Hinrichsen and Pritchard (2005)].

Let $G \in \mathbb{C}^{N \times M}$ and denote its singular values in decreasing order with $\sigma_{1}(G) \geq \sigma_{2}(G) \geq \ldots$. A classical result from linear algebra and robust control theory, which lays the basis for the celebrated small gain theorem, relates the largest singular value of $G$ to the solutions of the equation

$$
\operatorname{det}(I+G \Delta)=0
$$

in the following way:

$\sigma_{1}(G)=\left\{\begin{array}{l}0, \text { if } \operatorname{det}(I+G \Delta) \neq 0, \forall \Delta \in \mathbb{C}^{M \times N}, \\ m_{u}^{-1}, \text { otherwise, }\end{array}\right.$

where

$m_{u}:=\min \left\{\sigma_{1}(\Delta): \Delta \in \mathbb{C}^{M \times N}\right.$ and $\left.\operatorname{det}(I+G \Delta)=0\right\}$.

We refer to $\Delta$ as the 'uncertainty', as in a robust control framework, (A.1) typically originates from a feedback interconnection of a nominal transfer function and an uncertainty block.

Next we reconsider the solutions of equation (A.1), where $\Delta$ is restricted to having a particular structure by imposing $\Delta \in \Delta$, with $\boldsymbol{\Delta}$ a closed subset of $\mathbb{C}^{M \times N}$. In analogy with (A.2) one defines the structured singular value of the matrix $G$ with respect to the uncertainty set $\Delta$ as

$$
\mu_{\Delta}(G):=\left\{\begin{array}{l}
0, \text { if } \operatorname{det}(I+G \Delta) \neq 0, \forall \Delta \in \boldsymbol{\Delta}, \\
m_{s}^{-1}, \text { otherwise. }
\end{array}\right.
$$

where

$$
m_{s}=\min \left\{\sigma_{1}(\Delta): \Delta \in \boldsymbol{\Delta} \text { and } \operatorname{det}(I+G \Delta)=0\right\} .
$$

It directly follows from the definition that

$$
\mu_{\Delta}(G) \leq \sigma_{1}(G) .
$$

Furthermore, if $\mathbb{C} \Delta=\Delta$, then

$$
\mu_{\boldsymbol{\Delta}}(G)=\max _{\Delta \in \boldsymbol{\Delta}, \sigma_{1}(\Delta)=1} r_{\sigma}(G \Delta),
$$

with $r_{\sigma}(\cdot)$ the spectral radius.

In what follows we restrict ourselves for simplicity to an uncertainty set $\Delta$ of the form

$$
\begin{gathered}
\boldsymbol{\Delta}:=\left\{\operatorname{diag}\left(\Delta_{0}, \ldots, \Delta_{f}, d_{0} I_{m_{0}}, \ldots, d_{s} I_{m_{s}}\right):\right. \\
\Delta_{i} \in \mathbb{C}^{k_{i} \times l_{i}}, d_{j} \in \mathbb{C} \\
0 \leq i \leq f, 0 \leq j \leq s\}
\end{gathered}
$$

where $\operatorname{diag}(\cdot)$ represents a block diagonal matrix, $\sum_{i=0}^{f} k_{i}+\sum_{i=0}^{s} m_{i}=M$ and $\sum_{i=0}^{f} l_{i}+\sum_{i=0}^{s} m_{i}=$ $N$. Such a set satisfies $\mathbb{C} \boldsymbol{\Delta}=\boldsymbol{\Delta}$. Furthermore, based on a slight generalization of [(Packard and Doyle,
1993, Lemma 6.3)] to non-square block diagonal perturbations, the search space of the optimization in the right hand side of (A.5) can be restricted. This results in

$$
\mu_{\Delta}(G)=\max _{U \in \mathcal{U}} r_{\sigma}(G U),
$$

where $\mathcal{U} \subseteq \Delta$ is defined as

$$
\begin{aligned}
\mathcal{U}:= & \left\{\operatorname{diag}\left(U_{0}, \ldots, U_{f}, u_{0} I_{m_{0}}, \ldots, u_{s} I_{m_{s}}\right):\right. \\
& U_{i} \in \mathbb{C}^{k_{i} \times l_{i}}, u_{j} \in \mathbb{C}, \sigma_{k}\left(U_{i}\right)=1, \\
& 1 \leq k \leq \min \left(k_{i}, l_{i}\right),\left|u_{j}\right|=1, \\
& 0 \leq i \leq f, 0 \leq j \leq s\} .
\end{aligned}
$$

Note that the elements of $\mathcal{U}$ are unitary matrices if the uncertainty structure only involves square blocks, that is, $k_{i}=l_{i}, i=1, \ldots, f$.

Next, the following invariance property can easily be checked:

$$
\mu_{\boldsymbol{\Delta}}(G)=\mu_{\boldsymbol{\Delta}}\left(D_{2} G D_{1}^{-1}\right), \forall\left(D_{1}, D_{2}\right) \in \mathcal{D},
$$

where

$$
\begin{aligned}
\mathcal{D}:=\{ & \left(D_{1}, D_{2}\right): \\
& D_{1}=\operatorname{diag}\left(a_{0} I_{k_{1}}, \ldots, a_{f} I_{k_{f}}, D_{0}, \ldots, D_{s}\right), \\
& D_{2}=\operatorname{diag}\left(a_{0} I_{l_{1}}, \ldots, a_{f} I_{l_{f}}, D_{0}, \ldots, D_{s}\right): \\
& \left.a_{i}>0, D_{i} \in \mathbb{C}^{m_{i} \times m_{i}}, D_{i}^{*}=D_{i}>0\right\} .
\end{aligned}
$$

From (A.7) and the combination of (A.8) and (A.4) we finally obtain

$\max _{U \in \mathcal{U}} r_{\sigma}(G U)=\mu_{\Delta}(G) \leq \min _{\left(D_{1}, D_{2}\right) \in \mathcal{D}} \sigma_{1}\left(D_{2} G D_{1}^{-1}\right)$.

Therefore, optimization procedures are typically used to compute estimates for $\mu_{\boldsymbol{\Delta}}(G)$. The function $U \in$ $\mathcal{U} \rightarrow r_{\sigma}(G U)$ may have several local maxima and, for this, a local search for a maximum is not guaranteed to lead to $\mu_{\boldsymbol{\Delta}}(G)$, but to lower bounds. An appropriate formulation of the optimality condition enables algorithms which resemble power algorithms for computing eigenvalues and singular values, see Ref. [Packard et al. (1988)] for an example. Although the convergence of such algorithms to $\mu_{\Delta}(G)$ is not guaranteed either and they may converge to values corresponding to lower bounds on $\mu_{\Delta}(G)$, they have proven their effectiveness in practise. The computation of the upperbound in (A.9) can be recast into a standard convex optimization problem. However, in general $\mu_{\Delta}(G)$ is not equal to the upper-bound. An exception to this holds if the number of blocks in the matrices belonging to the uncertainty set $\boldsymbol{\Delta}$ satisfies $f+2 s \leq 3$ and all the blocks are square, $k_{i}=l_{i}, i=0, \ldots, f$. 